\title{
EFFECTS OF THE FIBERS' SHAPE AND VOLUME FRACTION ON THE STRENGTH OF IDEALLY PLASTIC FIBER REINFORCED COMPOSITES*
}

\author{
GUILLERMO H. GOLDSZTEIN ${ }^{\dagger}$
}

\begin{abstract}
We consider fiber reinforced composites where both the matrix and the fibers are ideally plastic materials. We restrict our attention to microstructures and applied stresses that lead to both microscopic and macroscopic antiplane shear deformations. We describe a bound we have recently obtained on the yield set of the composite in terms of the shape of the fibers, their volume fraction, and the yield set of the matrix. We construct examples of composites showing that our bound is essentially optimal.
\end{abstract}

Key words. ideal plasticity, fiber reinforced composites, homogenization, translation method, antiplane shear

AMS subject classifications. 74Q05, 74Q15, 74Q20

DOI. $10.1137 / 110827193$

1. Introduction. The stresses that an ideally plastic material can withstand form a bounded closed set $\overline{\mathcal{Y}}$ in the space of symmetric $3 \times 3$ real matrices. The set $\overline{\mathcal{Y}}$, which is a material property, is called the yield set or strength domain. Unlike brittle materials, ideally plastic materials do not break. When subjected to a stress that is in the boundary of $\overline{\mathcal{Y}}$, the material experiences a permanent deformation, usually called plastic deformation.

Fiber reinforced composites are materials made of solid fibers embedded in a weaker solid referred to as the matrix. We consider in this paper fiber reinforced composites where all the fibers are made of the same ideally plastic material. We denote by $\overline{\mathcal{Y}}_{\mathrm{f}}$ the yield set of the material the fibers are made of. We also assume that the matrix is made of an ideally plastic solid with a different yield set $\overline{\mathcal{Y}}_{\mathrm{m}}$. Thus, the stresses within the fibers are restricted to the set $\overline{\mathcal{Y}}_{\mathrm{f}}$, but within the matrix the stresses are restricted to the set $\overline{\mathcal{Y}}_{\mathrm{m}}$. More precisely, we denote by $\Omega$ the region in space occupied by the composite; for each $\mathbf{x} \in \Omega$, we define

$$
\overline{\mathcal{Y}}_{\mathbf{x}}= \begin{cases}\overline{\mathcal{Y}}_{\mathrm{f}} & \text { if } \mathbf{x} \text { is in a fiber } \\ \overline{\mathcal{Y}}_{\mathrm{m}} & \text { if } \mathbf{x} \text { is in the matrix. }\end{cases}
$$

Then, if the composite is subject to a stress $\bar{\sigma}$ that may vary within the material $\bar{\sigma}=\bar{\sigma}(\mathbf{x})$, we have

$$
\bar{\sigma}(\mathbf{x}) \in \overline{\mathcal{Y}}_{\mathbf{x}}
$$

for all $\mathbf{x} \in \Omega$. We will only consider time independent stresses $\bar{\sigma}$, and thus $\bar{\sigma}$ also satisfies the equilibrium equations

$$
\nabla \cdot \bar{\sigma}=0
$$

where $\nabla \cdot \bar{\sigma}$ is the divergence of $\bar{\sigma}$.

\footnotetext{
${ }^{*}$ Received by the editors March 10, 2011; accepted for publication (in revised form) March 2, 2012; published electronically May 17, 2012.

http://www.siam.org/journals/siap/72-3/82719.html

†School of Mathematics, Georgia Institute of Technology, Atlanta, GA 30332-0160 (ggold@math. gatech.edu).
} 
Loosely speaking, the microstructure or microgeometry of the composite refers to the description of the regions in space occupied by the fibers and the matrix. More precisely, the microstructure is determined by the function $\mathbf{x} \mapsto \overline{\mathcal{Y}}_{\mathbf{x}}$.

Assume the microstructure $\overline{\mathcal{Y}}_{\mathrm{x}}$ is periodic with period cell $\bar{Q}$, where $\bar{Q}$ is a parallelepiped. A stress field $\bar{\sigma}$ is said to be admissible if it is $\bar{Q}$-periodic and satisfies the restrictions (1.2) and the equilibrium equations (1.3). In the limit in which the size of the period cell $\bar{Q}$ is much smaller than the size of the material $\Omega$, the composite behaves macroscopically as a homogeneous material that can withstand only the stresses that belong to the set $\overline{\mathcal{Y}}_{\text {hom }}$ defined by

$$
\overline{\mathcal{Y}}_{\text {hom }}=\{\bar{\tau}: \bar{\tau}=\langle\bar{\sigma}\rangle \text { for some } \bar{\sigma} \text { admissible }\},
$$

where $\langle\bar{\sigma}\rangle$ is the average of $\bar{\sigma}$, i.e., $\langle\bar{\sigma}\rangle=|\bar{Q}|^{-1} \int_{\bar{Q}} \bar{\sigma}(\mathbf{x}) \mathrm{d} \mathbf{x}$ with $|\bar{Q}|$ being the volume of $\bar{Q}$. In other words, $\overline{\mathcal{Y}}_{\text {hom }}$ is the set of macroscopic stresses that the composite can withstand. We will refer to $\overline{\mathcal{Y}}_{\text {hom }}$ as the yield set of the composite. The justification of (1.4) is a well-understood fact of the theory of homogenization. We refer the reader to $[4,7,10,30,53,59,60,61]$ for more details.

In practice, all the details of the microstructure $\overline{\mathcal{Y}}_{\mathrm{x}}$ are not known or cannot be controlled. Instead, only some partial information, such as the volume fraction of the fibers, is available. Accordingly, the general objective in the mathematical study of these types of materials has been to obtain bounds or estimates on $\overline{\mathcal{Y}}_{\text {hom }}$, the set of stresses the composite can withstand, in terms of the yield sets of the matrix and the fibers, and information that may be available about the microstructure. This is also our goal in this paper.

Fiber reinforced composites, where both the fibers and the matrix are (to a good approximation) ideally plastic, are very important and widely used in applications. Thus, their study, both theoretical and experimental, is a very active field of research.

From a mathematical point of view, fiber reinforced composites belong to the wider class of materials known as heterogeneous solids, which include composites and polycrystals. The same mathematical tools are sometimes used to study different heterogeneous solids. Ideally plastic materials are highly nonlinear. Nevertheless, heterogeneous materials made of ideally plastic pure phases (the fibers and the matrix in our case) have proved to be amenable to detailed mathematical analysis. Thus, their study is very appealing since it can serve to test the existing mathematical methods, to develop new ones, and to gain intuition on the behavior of real materials. For the reasons mentioned in this and the previous paragraphs, the literature on the mathematical study of heterogeneous materials made of ideally plastic pure phases is very extensive. Some examples include $[1,2,3,13,14,15,16,51,18,19,21,22$, $23,25,28,29,56,43,44,45,46,48,49,54,62,67]$. Examples of related nonlinear homogenization problems include $[42,64,68]$.

In our work, we will study the effect of the fiber shape on the yield set of the composite in the scalar two-dimensional problem that results from restricting our attention to applied stresses and microstructures that lead to both microscopic and macroscopic antiplane shear. More precisely, after we describe a bound we have recently obtained on the yield set of the composite [20], we will construct examples of composites showing that our bound is essentially optimal. We stress that the contribution of this paper is not the bound described in section 3 , as the derivation of this bound has already been published [20]. The contribution of this paper is to show that this bound is in fact sharp.

We mention that, while our results are completely new, the yield set of fiber or 
particle reinforced composites has been studied $[26,5,8,9,6,11,12,24,33,27,31$, $32,34,35,36,37,38,47,50,52,55,57,58,63]$.

Our bound can be considered to be an application of a mathematical technique known as the translation method $[41,65,66]$. Other examples where the translation method was used to study nonlinear composites or polycrystals include [17, 29, 18, 39, 40]. Particularly, the work in [29] has motivated a lot of work in this area of research.

This paper is organized as follows: In section 2 we reduce the original threedimensional vectorial problem to a two-dimensional scalar problem by considering applied stresses and microstructures that lead to both microscopic and macroscopic antiplane shear. In section 3 we describe our bound on the strength of the weakest direction of the composites we consider. In section 4 we analyze the case when the cross section of the fibers are circular. In section 5 we study composites with fibers with slender cross sections. In section 6 we show that our bound (in a sense specified in that section) is sharp. We conclude with some discussions in section 7 .

2. The microstructures and antiplane shear. As usual, we denote by $x_{1}, x_{2}$, and $x_{3}$ the components of the position vector $\mathbf{x}$, i.e., $\mathbf{x}=\left(x_{1}, x_{2}, x_{3}\right)$. We assume that the fibers are parallel to the $x_{3}$-axis and the cross sections of the fibers (intersection of a fiber with a plane of the form $x_{3}=$ constant) are independent of $x_{3}$. In other words, the function $\mathbf{x} \mapsto \overline{\mathcal{Y}}_{\mathbf{x}}$ is independent of $x_{3}$. Note that, as a consequence, the period cell $\bar{Q}$ is of the form $\bar{Q}=Q \times[a, b]$, where $Q$ is a parallelogram in $\mathbb{R}^{2}$ and $a$ and $b$ are any numbers that satisfy $a<b$.

We also assume that the yield sets $\overline{\mathcal{Y}}_{\mathrm{f}}$ and $\overline{\mathcal{Y}}_{\mathrm{m}}$ are convex sets with the following symmetric property: For both $\overline{\mathcal{Y}}=\overline{\mathcal{Y}}_{\mathrm{f}}$ and $\overline{\mathcal{Y}}=\overline{\mathcal{Y}}_{\mathrm{m}}$,

$$
\text { if }\left[\begin{array}{lll}
\bar{\sigma}_{11} & \bar{\sigma}_{12} & \bar{\sigma}_{13} \\
\bar{\sigma}_{21} & \bar{\sigma}_{22} & \bar{\sigma}_{23} \\
\bar{\sigma}_{31} & \bar{\sigma}_{32} & \bar{\sigma}_{33}
\end{array}\right] \text { belongs to } \overline{\mathcal{Y}}, \text { so does }\left[\begin{array}{rrr}
-\bar{\sigma}_{11} & -\bar{\sigma}_{12} & \bar{\sigma}_{13} \\
-\bar{\sigma}_{21} & -\bar{\sigma}_{22} & \bar{\sigma}_{23} \\
\bar{\sigma}_{31} & \bar{\sigma}_{32} & -\bar{\sigma}_{33}
\end{array}\right] \text {. }
$$

This assumption is not restrictive at all. We refer the reader to [29] and the references therein for a discussion of this symmetry condition.

Due to our assumptions, the two-dimensional scalar problem that corresponds to antiplane shear is well defined. More precisely, for $i=\mathrm{f}, i=\mathrm{m}$, and $i=\mathrm{hom}$, we define the sets

$$
\mathcal{Y}_{i}=\left\{\sigma=\left(\sigma_{1}, \sigma_{2}\right) \in \mathbb{R}^{2}:\left[\begin{array}{rrr}
0 & 0 & \sigma_{1} \\
0 & 0 & \sigma_{2} \\
\sigma_{1} & \sigma_{2} & 0
\end{array}\right] \in \overline{\mathcal{Y}}_{i}\right\} .
$$

For each $\mathbf{x}=\left(x_{1}, x_{2}\right) \in \mathbb{R}^{2}$, we also define

$$
\mathcal{Y}_{\mathbf{x}}= \begin{cases}\mathcal{Y}_{\mathrm{f}} & \text { if }\{\mathbf{x}\} \times \mathbb{R} \text { is included in a fiber, } \\ \mathcal{Y}_{\mathrm{m}} & \text { if }\{\mathbf{x}\} \times \mathbb{R} \text { is included in the matrix. }\end{cases}
$$

We say that a two-dimensional vector field $\sigma=\sigma(\mathbf{x})=\left(\sigma_{1}\left(x_{1}, x_{2}\right), \sigma_{2}\left(x_{1}, x_{2}\right)\right)$ is admissible if it is $Q$-periodic, it satisfies the restrictions

$$
\sigma(\mathbf{x}) \in \mathcal{Y}_{\mathbf{x}} \text { for all } \mathbf{x} \in \mathbb{R}^{2},
$$

and the equilibrium equations

$$
\nabla \cdot \sigma=0
$$

where $\nabla \cdot \sigma$ is the divergence of $\sigma$ in two dimensions. 
It can be easily shown that, given the conditions stated in this section,

$$
\mathcal{Y}_{\text {hom }}=\{\tau: \tau=\langle\sigma\rangle \text { for some two-dimensional admissible vector field } \sigma\},
$$

where $\langle\sigma\rangle$ is now the two-dimensional average of $\sigma$, i.e., $\langle\sigma\rangle=|Q|^{-1} \int_{Q} \sigma(\mathbf{x}) \mathrm{d} \mathbf{x}$ with $|Q|$ being the area of $Q$.

All the vector fields we will consider in the rest of this paper are two-dimensional. The two-dimensional sets $\mathcal{Y}_{\mathrm{f}}, \mathcal{Y}_{\mathrm{m}}$, and $\mathcal{Y}_{\text {hom }}$ will also be referred to as the yield sets of the fibers, the matrix, and the composite, respectively.

We will assume that the sets $\mathcal{Y}_{\mathrm{f}}$ and $\mathcal{Y}_{\mathrm{m}}$ are circles centered at the origin with radii $Y_{\mathrm{f}}$ and $Y_{\mathrm{m}}$, respectively. Thus, defining

$$
Y_{\mathbf{x}}= \begin{cases}Y_{\mathrm{f}} & \text { if }\{\mathbf{x}\} \times \mathbb{R} \text { is included in a fiber, } \\ Y_{\mathrm{m}} & \text { if }\{\mathbf{x}\} \times \mathbb{R} \text { is included in the matrix, }\end{cases}
$$

we have that a vector field $\sigma$ is admissible if and only if $\sigma$ is $Q$-periodic, $\|\sigma(\mathbf{x})\| \leq Y_{\mathbf{x}}$ for all $\mathbf{x} \in \mathbb{R}^{2}$, and $\nabla \cdot \sigma=0$, where $\|\cdot\|$ denotes the euclidean norm, i.e., $\left\|\left(z_{1}, z_{2}\right)\right\|=$ $\sqrt{z_{1}^{2}+z_{2}^{2}}$ for all $\mathbf{z}=\left(z_{1}, z_{2}\right) \in \mathbb{R}^{2}$. Note that divergence-free vector fields can be regarded as the velocity field of an incompressible fluid. Thus, in the rest of this paper, we will sometimes refer to two-dimensional divergence-free vector fields as stresses and sometimes as fluid velocity fields.

3. Bound on the weakest direction of the yield set of the composite. We will refer to the cross sections of the fibers as the inclusions. In other words, the inclusions are the connected components of the set $\left\{\mathbf{x} \in \mathbb{R}^{2}: Y_{\mathbf{x}}=Y_{\mathrm{f}}\right\}$.

For any inclusion $I$, we will assume in the rest of this paper that the number of points that belong to the boundary of $I$ and also to the boundary of any other inclusion is finite.

We denote by $\nu$ the volume fraction of the inclusions, i.e.,

$$
\nu=\frac{\mid\{\mathbf{x} \in Q \text { and } \mathbf{x} \in \text { an inclusion }\} \mid}{|Q|} .
$$

For each inclusion $I$ we denote by $P_{I}$ its perimeter, i.e., $P_{I}$ is the length of the boundary of $I$. We define the parameter

$$
\eta=\max _{I \text { inclusion }} \frac{P_{I}^{2}}{|I|} .
$$

When reinforcing a material, it is usually desirable that the material is strong in all directions. Thus, it is of interest to evaluate the strength of the weakest direction of the composite. Our bound will in fact be a bound on the strength of the weakest direction of the composite (the word strength here is used in an informal way). This motivates the following definition. For any positive number $Y$, we denote by $B_{Y}$ the open ball of radius $Y$ centered at the origin of $\mathbb{R}^{2}$. We define

$$
Y_{\text {hom }}^{\text {weak }}=\max _{\left\{Y: B Y \subseteq \mathcal{Y}_{\text {hom }}\right\}} Y \text {. }
$$

Recall that $\mathcal{Y}_{\text {hom }}$ is, in this two-dimensional context, the set of stresses the composite can withstand. A little thought will convince the reader that $Y_{\text {hom }}^{\text {weak }}$ is the strength of the weakest direction of the composite. The composite can withstand all stresses of 
norm less than $Y_{\text {hom }}^{\text {weak }}$. On the other hand, if $Y>Y_{\text {hom }}^{\text {weak }}$, there are stresses of norm $Y$ that the composite cannot withstand.

We have recently obtained the following bound [20].

THEOREM 3.1. The strength of the weakest direction of the composite, $Y_{\mathrm{hom}}^{\mathrm{weak}}$, satisfies the following bound:

$$
Y_{\text {hom }}^{\text {weak }} \leq Y_{\mathrm{m}} \sqrt{(1-\nu)+\frac{1}{2} \nu \eta}
$$

where $\nu$ is the volume fraction of the inclusions and $\eta$ is as defined in (3.2).

4. Composites with circular inclusions. The square of the perimeter of a circle divided by its area is equal to $4 \pi$. Thus,

$$
\eta=4 \pi \quad \text { if all the inclusions are circular, }
$$

where $\eta$ is the parameter defined in (3.2). As a consequence, our bound (equation (3.4)) becomes

$$
Y_{\text {hom }}^{\text {weak }} \leq Y_{\mathrm{m}} \sqrt{(1-\nu)+2 \nu \pi} \quad \text { if all the inclusions are circular, }
$$

where, as before, $\nu$ is the volume fraction of the inclusions. In particular, since $0<\nu<1$, we have that

$$
Y_{\text {hom }}^{\text {weak }}<Y_{\mathrm{m}} \sqrt{2 \pi} \quad \text { if all the inclusions are circular. }
$$

Note that, no matter the strength of the inclusions, if they are circular, the strength of the composite is never more than $\sqrt{2 \pi}$ times the strength of the matrix (again the word strength is used informally, but its meaning in this context should be clear). We remark that the fact that the yield set of a composite with circular inclusions is bounded independently of the yield set of the inclusions is not our discovery but is a well-known fact (see [47, 63] for example).

5. Composites with slender rectangular inclusions. Our bound suggests that, to obtain strong composites, we should use inclusions that lead to a large parameter $\eta$. As illustrated in the next example, $\eta$ becomes large when the inclusions are slender.

A natural choice would be to consider elliptical inclusions. However, to make the calculations easier to follow, we will assume the inclusions to be rectangular. For each inclusion $I$, let $\gamma_{I}$ be the length of one of the longest sides of $I$ divided by the length of one of the shortest sides of $I$. Assume that $\gamma_{I}$ is the same value for all inclusions $I$. Denote this value by $\gamma$, i.e., $\gamma=\gamma_{I}$ for any inclusion $I$,

$$
\gamma=\frac{\text { length of longest side of an inclusion }}{\text { length of shortest side of the same inclusion }} .
$$

Note that the square of the perimeter of one of these rectangular inclusions divided by its area is equal to $4(\gamma+1)^{2} / \gamma$,

$$
\eta=\frac{4(\gamma+1)^{2}}{\gamma}
$$

where $\eta$ is the parameter defined in (3.2). 
To be under the assumptions of our analysis (section 3), we assume that the boundary of two different inclusions intersect at most at one point. We are particularly interested in the limit $\gamma \gg 1$. Note that $\eta \approx 4 \gamma$ for $\gamma \gg 1$. As a consequence, our bound (equation (3.4)) becomes

$$
Y_{\text {hom }}^{\text {weak }} \leq Y_{\mathrm{m}} \sqrt{2 \nu \gamma} \text { for } \gamma \gg 1 \text { and } \nu>0,
$$

where, as before, $\nu$ is the volume fraction of the inclusions.

The natural question that our analysis raises is whether the bound of (5.3) is sharp or not. To show that the answer to this question is yes, we next construct a composite with rectangular inclusions whose weakest direction is of the order of $Y_{\mathrm{m}} \sqrt{\nu \gamma}$.

6. Composite with slender rectangular inclusions that is strong in all directions. We start by considering the composite of Figure 6.1. The inclusions are shaded and the matrix is white. Each inclusion is a rectangle of width $w$ and length $\ell$, with $w \ll \ell$. The inclusions are embedded in the matrix forming the regular pattern shown in Figure 6.1. We denote by $\varepsilon$ the distance between neighboring inclusions. We assume that $\varepsilon \ll w$.

We now construct an admissible field $\sigma$ that satisfies $\langle\sigma\rangle \approx\left(Y_{\mathrm{m}} \ell /(2 w), 0\right)$. Note that, as mentioned before, $\sigma$ can be regarded as the velocity field of an incompressible fluid. In this context, the fact that $Y_{\mathrm{m}} \ll Y_{\mathrm{f}}$ means that fluid can flow much faster within the inclusions than in the matrix. In Figure 6.1 (left), the dashed lines with arrows denote the direction of $\sigma$. Roughly speaking, fluid flows quickly in the horizontal direction within the inclusions, and fluid flows slowly in the vertical direction as fluid needs to go through the matrix as it flows from one inclusion to a neighboring inclusion. The details of the flow are shown in Figure 6.1 (right), where we only show one full inclusion and a quarter of each of its neighboring inclusions. In the regions where fluid flows horizontally, $\sigma=\left(Y_{\mathrm{m}}(\ell-\varepsilon) / w, 0\right)$. In the regions where fluid flows vertically, $\sigma=\left(0, \pm Y_{\mathrm{m}}\right)$. Note that $\sigma=0$ inside the vertical rectangles of Figure 6.1 (left) that are included in the matrix. It can be calculated that $\langle\sigma\rangle=\left(Y_{\mathrm{m}}(\ell-\varepsilon) /(2 w+2 \varepsilon), 0\right)$, and thus, neglecting $\varepsilon$, we get that

$$
\text { average velocity of the flow in Figure } 6.1 \approx Y_{\mathrm{m}} \frac{\gamma}{2}(1,0),
$$

where, as defined in (5.1), $\gamma=w / \ell$. Note that we have implicitly assumed that $Y_{\mathrm{f}} \geq Y_{\mathrm{m}} \gamma$.

In Figure 6.2 we show a rectangular stripe of length $L$ and width $W$ made of the composite of Figure 6.1, where the dimensions of the stripe are much bigger than the dimensions of the inclusions $\ell \ll L$ and $w \ll W$, and the longest side of the inclusions
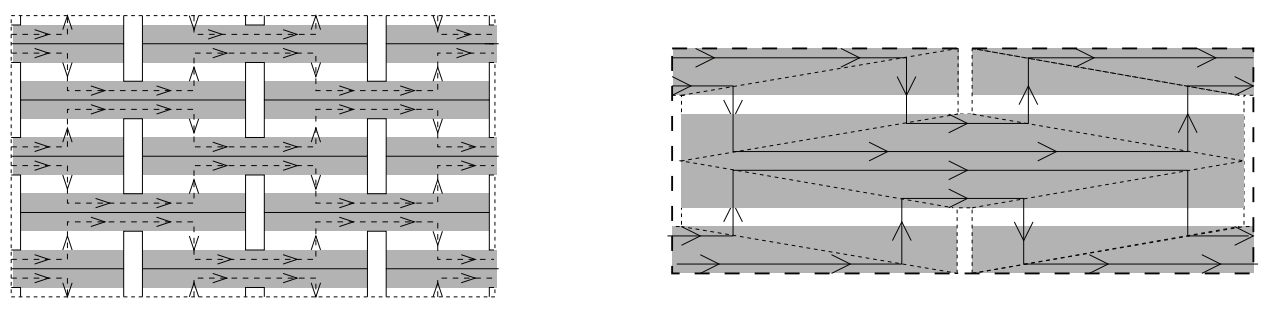

FIG. 6.1. Composite with rectangular inclusions and an admissible field $\sigma$ described in the text. 

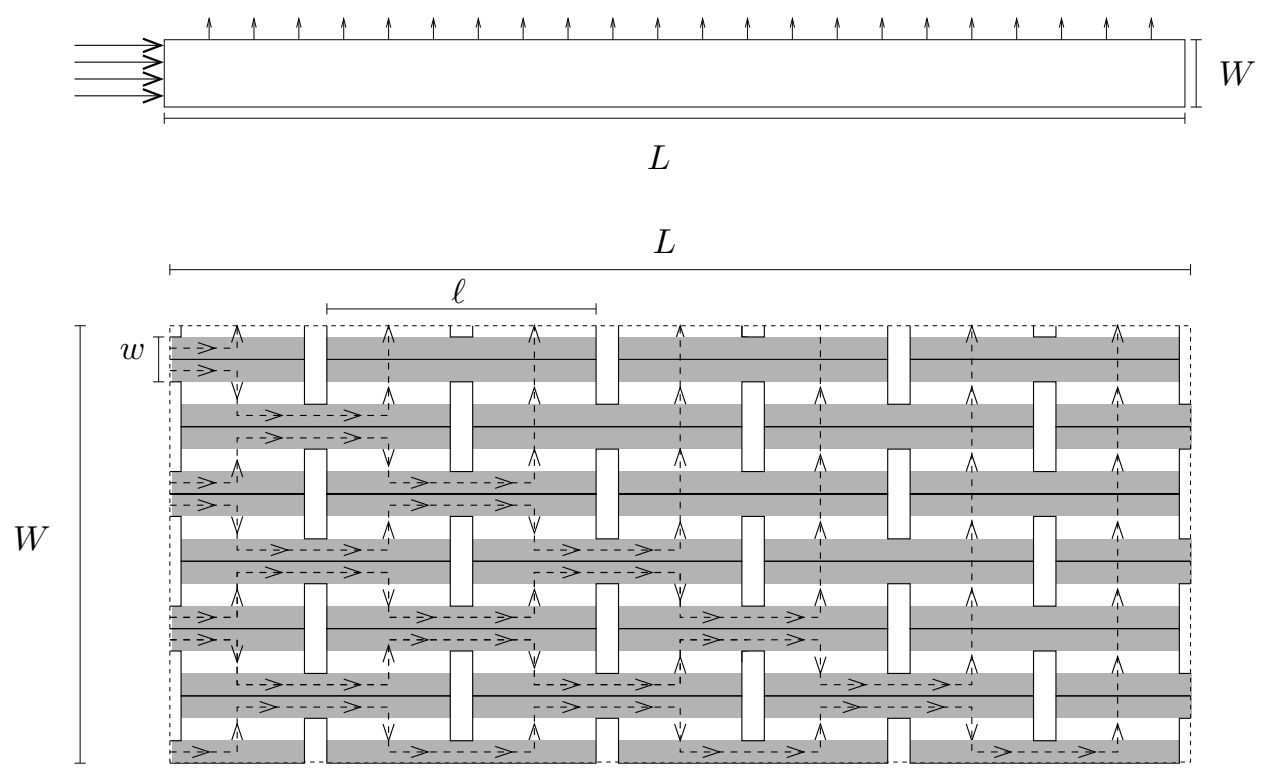

FIG. 6.2. Stripe made of the composite of Figure 6.1. Top figure, macroscopic view. Bottom figure, microscopic view. The arrows indicate the velocity field $\sigma$.

are parallel to the longest sides of the stripe. More precisely, $W=(2 n+1 / 2)(w+\varepsilon)$ and $L=(n+1 / 2)(\ell+\varepsilon)$, where we assume $n \gg 1$. Thus,

$$
\frac{L}{W} \approx \frac{\gamma}{2}
$$

where, as defined in (5.1), $\gamma=w / \ell$. In the example of Figure 6.2, $n=3$. Following similar arguments as in the construction of the flow in Figure 6.1, we construct, in Figure 6.2 , a new fluid velocity field, which we also call $\sigma$, that satisfies the constraints (2.4) and (2.5) such that fluid enters the stripe from the left side, fluid leaves the stripe from the top side, and no fluid crosses the other two sides. The rate at which fluid enters the stripe in Figure 6.2 is the same as if the flow were as in Figure 6.1. Thus, from (6.1), we have

$$
\text { rate at which area of fluid enters the stripe in Figure } 6.2 \approx Y_{\mathrm{m}} W \frac{\gamma}{2} \text {. }
$$

In Figure 6.3 we construct a periodic composite. That figure shows a period cell. The stripes seen in Figure 6.3 are as in Figure 6.2, but instead of being straight rectangles, these stripes are curved. Note that all of these stripes are finite in length. Note also that the inclusions are much smaller than the stripes and cannot be seen in Figure 6.3. To help the reader visualize this microstructure, we have shaded one of the stripes black.

Our goal final is to show that, for the composite of Figure 6.3, we can construct admissible fields $\sigma$ that satisfy $\langle\sigma\rangle \approx\left(Y_{\mathrm{m}} / 2\right) \sqrt{\gamma \nu / 2}( \pm 1, \pm 1)$, where as mentioned before $\gamma=\ell / w$. This and the fact that $\mathcal{Y}_{\text {hom }}$ is convex implies that, for the composite of Figure 6.3,

$$
\mathcal{Y}_{\text {hom }} \supseteq \text { ball centered at the origin of radius } \frac{Y_{\mathrm{m}}}{2} \sqrt{\frac{\gamma \nu}{2}} .
$$






FIG. 6.3. Periodic composite. The stripes (both shaded and white) are as in Figure 6.2, but curved.

As a consequence, for the composite of Figure 6.3, we have

$$
Y_{\text {hom }}^{\text {weak }} \geq Y_{\mathrm{m}} \sqrt{\frac{\gamma \nu}{8}} \text { for } \gamma \gg 1
$$

(see (3.3) for the definition of $Y_{\text {hom }}^{\text {weak }}$ ). This shows that our bound, which is (5.3) for composites with rectangular inclusions, gives the correct scaling law in terms of the parameters $Y_{\mathrm{m}}, \gamma$, and $\nu$.

We now proceed with the construction of the mentioned admissible fields. More precisely, we will construct an admissible field $\sigma$ such that $\langle\sigma\rangle \approx\left(Y_{\mathrm{m}} / 2\right) \sqrt{\gamma \nu / 2}(1,1)$ (the other fields with average $\left(Y_{\mathrm{m}} / 2\right) \sqrt{\gamma \nu / 2}( \pm 1, \pm 1)$ are constructed similarly).

To avoid a very crowded figure, in Figure 6.4 (left) we only show two half stripes. These two half stripes are part of the stripes in the upper left corner of Figure 6.3. Even though the stripes are not rectangles, since the bending of the stripes occurs in a length scale much larger than the length of the inclusions (which are not seen in this figure), we can use similar arguments as in Figure 6.2 to construct the flow displayed in Figure 6.4 (left). The arrows indicate the average direction of the flow when the flow is looked at in the scale of the stripes. If we were to look at the details of the flow in the scale of the inclusions, the flow would look like Figure 6.2 (bottom). Let $W$ be the width of a stripe and let $L$ now be the length of half a stripe; from the arguments in the discussion of Figure 6.3 , we need $W / L \leq 2 w / \ell=2 / \gamma$ so that this flow satisfies the constraints (2.4) and (2.5). Thus, we will have

(6.6) $\frac{L}{W} \approx \frac{\gamma}{2}$, where $L=$ length of half a stripe and $W=$ width of a stripe.

In Figure 6.4 (right), we show the complete upper left corner of Figure 6.3. The arrows indicate the average direction of $\sigma$. Fluid flows between neighboring stripes crossing the dashed lines. There is no flow across the solid lines. While we do not 

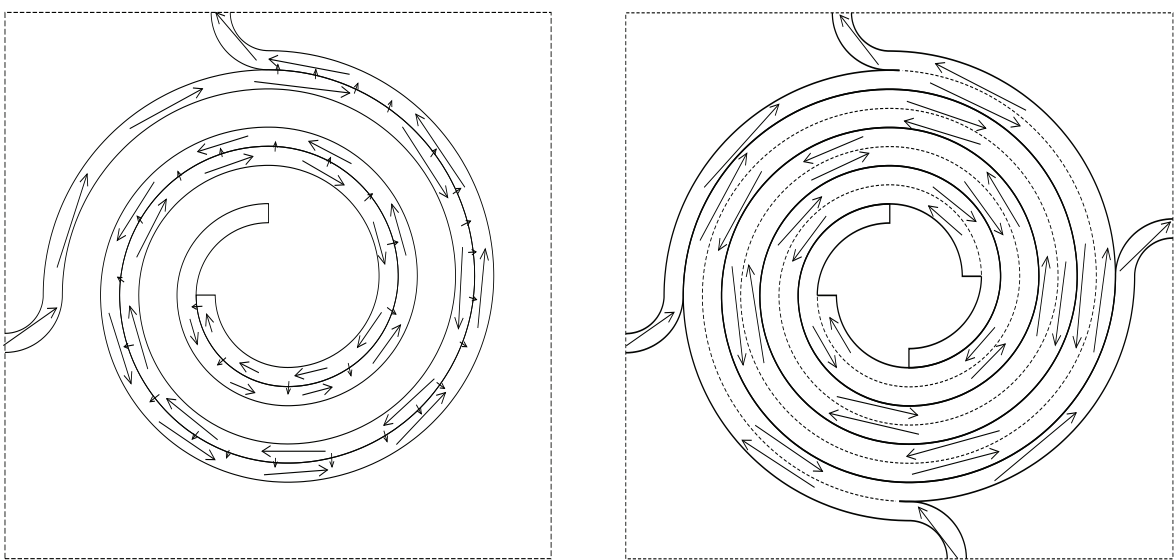

FIG. 6.4. Flow in the upper left corner of the composite of Figure 6.3. Only two half stripes are shown in the left figure. All of the stripes are shown in the right figure.

show it here, fluid flows similarly in the rest of the period cell. Fluid flows from stripe to a neighboring stripe so that, on average, fluid flows in the direction of the vector $(1,1)$.

We now compute $\langle\sigma\rangle$ for the flow of Figure 6.4. The period cell $Q$ is a square. Denote by $q$ the length of each side of $Q$. Simple arguments show that

$$
\langle\sigma\rangle=\frac{1}{q} \times \text { rate area of fluid enters } Q \text { from the left } \times(1,1) .
$$

Since fluid enters through the two stripes that intersect the left side of the period cell, (6.3) implies

$$
\langle\sigma\rangle \approx \frac{1}{q} Y_{\mathrm{m}} W \gamma(1,1),
$$

where we recall that $W$ is the width of each stripe. Recall also that $L$ is half the length of each stripe. In the limit in which the size of the inclusions is much smaller than the size of the stripes, we can fill most of the stripes with inclusions. Thus, since there are four half stripes in a quarter of the period cell, we have

$$
\nu \approx \frac{16 W L}{q^{2}} .
$$

Finally, (6.6), (6.8), and (6.9) imply

$$
\langle\sigma\rangle \approx Y_{\mathrm{m}} \sqrt{\frac{\nu \gamma}{8}}(1,1) .
$$

7. Discussion. In the two-dimensional scalar context of this paper, we have shown that a bound introduced in a previous work [20] is essentially optimal.

The effect of the inclusions in particle reinforced composites was also studied in $[34,35]$. In that work, the authors studied the effect of the shape of the inclusions. They considered the three-dimensional problem with inclusions being ellipsoids in which the lengths of two of the semiaxes are equal; i.e., they considered spheroid 
inclusions. They use a well-known and powerful variational method introduced in [44, $45]$ and obtained very interesting results.

Let $a, b$, and $c$ be the lengths of the semiaxes of the ellipsoid inclusions. In the work $[34,35]$, it is assumed that $a=b$. The particular case of aligned inclusions and the limit of $c \rightarrow \infty$, which the authors do consider, corresponds to fiber reinforced composites with circular cross sections. In this work, we are attempting to uncover the effect that the shape of cross sections has on the yield set of the composites, particularly slender cross sections. Thus, a comparison of our work with that of $[34,35]$ would be premature. However, it would be interesting to apply the variational method used in $[34,35]$ to the case when all three lengths of semiaxes are different $a<b<c$ and then take the limit $c \rightarrow \infty$. This corresponds to fiber reinforced composites with elliptical cross sections, and a detail comparison with the results of our work could be made.

\section{REFERENCES}

[1] M. Berveiller And A. Zaoui, An extension of the self consistent scheme to plastically-flowing polycrystals, J. Mech. Phys. Solids, 26 (1979), pp. 325-344.

[2] K. Bhattacharya And P. M. Suquet, A model problem concerning recoverable strains of shape-memory polycrystals, Proc. R. Soc. Lond. Ser. A Math. Phys. Eng. Sci., 461 (2005), pp. $2797-2816$.

[3] J. Bishop And R. Hill, A theory for the plastic distortion of a polycrystalline aggregate under combined stresses, Phil. Mag. A, 42 (1951), pp. 414-427.

[4] G. Bouchitté And P. Suquet, Homogenization, plasticity and yield design, in Composite Media and Homogenization, G. D. Maso and G. Dell'Antonio, eds., Birkhäuser, Basel, 1991, pp. 107-133.

[5] T. W. Butler AND E. J. Sullivan, JR., On the transverse strength of fiber reinforced materials, J. Appl. Mech., 40 (1973), pp. 523-526.

[6] P. DE BUHAN, Lower bound approach to the macroscopic strength properties of a soil reinforced by columns, C. R. Acad. Sci. Paris Sér. II, 317 (1993), pp. 287-293.

[7] P. DE Buhan And A. Taliercio, A homogenization approach to the yield strength of composite materials, Eur. J. Mech. A Solids, 10 (1991), pp. 129-154.

[8] G. DeBotton, The effective yield strength of fiber-reinforced composites, Internat. J. Solids Structures, 32 (1995), pp. 1743-1757.

[9] G. deBotton and P. Ponte Castaneda, Elastoplastic constitutive relations for fiber reinforced solids, Internat. J. Solids Structures, 30 (1993), pp. 1865-1890.

[10] F. Demengel and T. QI, Convex function of a measure obtained by homogenization, C. R. Acad. Sci. Paris Sér. I, 303 (1986), pp. 285-288.

[11] I. Doghri And C. Friebel, Effective elasto-plastic properties of inclusion-reinforced composites. Study of shape, orientation and cyclic response, Mech. Mater., 37 (2005), pp. 45-68.

[12] I. Doghri AND L. Tinel, Micromechanical modelling and computation of elasto-plastic materials reinforced with distributed-orientation fibers, Int. J. Plasticity, 21 (2005), pp. 1919-1940.

[13] D. C. DRUCKeR, The safety factor of an elastic-plastic body in plane strain, J. Appl. Mech., 18 (1951), pp. 371-378.

[14] D. C. Drucker, Extended limit design theorems for continuous media, Quart. Appl. Math., 9 (1952), pp. 381-389.

[15] D. C. Drucker, On minimum weight design and strength of non-homogeneous plastic bodies, in Non-homogeneity in Elasticity and Plasticity, W. Olszak, ed., Pergamon Press, New York, 1959.

[16] D. C. Drucker, Engineering and continuum aspects of high strength materials, in HighStrength Materials, V. F. Zackay, ed., Wiley, New York, 1965, pp. 795-833.

[17] A. Garroni And R. V. Kohn, Some three-dimensional problems related to dielectric breakdown and polycrystal plasticity, Proc. R. Soc. Lond. Ser. A Math. Phys. Eng. Sci., 459 (2003), pp. 2613-2625.

[18] G. H. Goldsztein, Rigid perfectly plastic two-dimensional polycrystals, Proc. R. Soc. Lond. Ser. A Math. Phys. Eng. Sci., 457 (2001), pp. 2789-2798.

[19] G. H. Goldsztein, Two-dimensional rigid polycrystals whose grains have one ductile direction, Proc. R. Soc. Lond. Ser. A Math. Phys. Eng. Sci., 459 (2003), pp. 1949-1989. 
[20] G. H. Goldsztein, Bound on the yield set of fiber reinforced composites subjected to antiplane shear, Discrete Contin. Dyn. Syst. Ser. B, 15 (2011), pp. 391-400.

[21] Z. HAshin, Failure criteria for unidirectional fiber composites, J. Appl. Mech., 47 (1980), pp. 329-334.

[22] R. HILl, A theory of the yielding and plastic flow of anisotropic metals, Proc. R. Soc. Lond. Ser. A Math. Phys. Eng. Sci., 193 (1948), pp. 281-297.

[23] R. HILL, Theory of mechanical properties of fibre-strengthened materials: II. Inelastic behavior, J. Mech. Phys. Solids, 12 (1964), pp. 213-218.

[24] G. Hu, A method of plasticity for general aligned spheroidal void or fiber-reinforced composites, Int. J. Plasticity, 12 (1996), pp. 439-449.

[25] W. Huang, Plastic behavior of some composite materials, J. Comp. Mat., 5 (1971), pp. 320338.

[26] J. W. Hutchinson, G. Bao, and R. M. McMeeking, Particle reinforcement of ductile matrices against plastic flow and creep, Acta Metall. Mater., 39 (1991), pp. 1871-1882.

[27] B. Ji And T. WANG, Plastic constitutive behavior of short-fiber/particle reinforced composites, Int. J. Plasticity, 19 (2003), pp. 565-581.

[28] U. F. Kocks, The relation between polycrystal deformation and single-crystal deformation, Metallurgical Trans., 1 (1970), pp. 1121-1143.

[29] R. V. Kohn and T. D. LitTle, Some model problems of polycrystal plasticity with deficient basic crystals, SIAM J. Appl. Math., 59 (1998), pp. 172-197.

[30] S. Kozlov, V. Jikov, AND O. Oleinik, Homogenization of Differential Operations and Integral Functionals, Springer-Verlag, New York, 1994.

[31] R. H. Lance and D. N. Robinson, A maximum shear stress theory of plastic failure of fibrereinforced materials, J. Mech. Phys. Solids, 19 (1971), pp. 49-60.

[32] B. J. LEE AND M. E. MEAR, On the yield strength of metals containing spheroidals inclusions or voids, Mech. Mater., 12 (1991), pp. 191-205.

[33] W. Li, D. Jeulin, and M. Ostoja-Starzewski, On the geodesic property of strain field patterns in elastoplastic composites, Proc. R. Soc. Lond. Ser. A Math. Phys. Eng. Sci., 464 (2008), pp. 1217-1227.

[34] G. Li and P. Ponte Castaneda, The effect of particle shape and stiffness on the constitutive behavior of metal-matrix composites, Internat. J. Solids Structures, 30 (1993), pp. 31893209.

[35] G. Li, P. Ponte Castaneda, and A. S. Douglas, Constitutive models for ductile solids reinforced by rigid spheroidal inclusions, Mech. Mater., 15 (1993), pp. 279-300.

[36] S. Majumdar and P. V. McLaughlin, JR., Upper bounds to in-plane shear strength of unidirectional fiber-reinforced composites, J. Appl. Mech., 40 (1973), pp. 824-825.

[37] S. Majumdar AND P. V. McLaughlin, JR., Effects of phase geometry and volume fraction on the plane stress limit analysis of a unidirectional fiber-reinforced composite, Internat. J. Solids Structures, 11 (1975), pp. 777-791.

[38] P. V. McLaughlin, Plastic limit behavior of filament-reinforced materials, Internat. J. Solids Structures, 8 (1972), pp. 1299-1318.

[39] G. W. Milton, On characterizing the set of possible tensors of composites. the variational method and the translation method, Comm. Pure Appl. Math., 43 (1990), pp. 63-125.

[40] G. W. Milton and S. K. Serkov, Bounding the current in nonlinear conducting composites, J. Mech. Phys. Solids, 48 (2000), pp. 1295-1324.

[41] F. Murat, Compacité par compensation: Condition necessaire et suffisante de continuité faible sous une hypothése de rang constant, Ann. Scuola Norm. Sup. Pisa Cl. Sci., 8 (1981), pp. 68-102.

[42] V. Nesi, A. Garroni, and M. Ponsiglione, Dielectric breakdown: Optimal bounds, Proc. R. Soc. Lond. Proc. Ser. A Math. Phys. Eng. Sci., 457 (2001), pp. 2317-2335.

[43] T. Olson, Improvements on Taylor's upper bound for rigid-plastic composites, Mater. Sci. Eng. A, 175 (1994), pp. 15-20.

[44] P. Ponte Castaneda, The effective mechanical properties of nonlinear isotropic composites, J. Mech. Phys. Solids, 39 (1991), pp. 45-71.

[45] P. Ponte Castaneda, New variational principles in plasticity and their applications to composite materials, J. Mech. Phys. Solids, 40 (1992), pp. 1757-1788.

[46] P. Ponte Castaneda, Exact second-order estimates for the effective mechanical properties of nonlinear composite materials, J. Mech. Phys. Solids, 44 (1996), pp. 827-862.

[47] P. Ponte Castaneda and G. DeBotton, On the homogenized yield strength of two-phase composites, Proc. R. Soc. Lond. Ser. A Math. Phys. Eng. Sci., 438 (1992), pp. 419-431.

[48] P. Ponte Castaneda and M. Nebozhyn, Variational estimates of the self consistent type for some model nonlinear polycrystals, Proc. R. Soc. Lond. Ser. A Math. Phys. Eng. Sci., 453 (1997), pp. 2715-2724.

Copyright $@$ ( ) by SIAM. Unauthorized reproduction of this article is prohibited. 
[49] P. Ponte Castaneda and P. Suquet, Nonlinear composites, Adv. Appl. Mech., 34 (1997), pp. 171-302.

[50] W. Prager, Plastic failure of fiber reinforced materials, J. Appl. Mech., 36 (1969), pp. 542-544.

[51] W. Prager, D. C. Drucker, and H. J. Greenberg, Extended limit design theorems for continuous media, Quart. Appl. Math., 9 (1952), pp. 381-389.

[52] Y. P. QIU AND G. J. WENG, The influence of inclusion shape on the overall behavior of a two-phase isotropic composite, Internat. J. Solids Structures, 27 (1991), pp. 1537-1550.

[53] K. SAB, Homogenization of non-linear random media by a duality method. Application to plasticity, Asymptot. Anal., 9 (1994), pp. 311-336.

[54] G. SACHS, Zur ableitung einer fleissbedingun, Z. Ver. Dtsch. Ing., 72 (1928), pp. 734-736.

[55] L. S. Shu And B. W. Rosen, Strength of fiber-reinforced composites by limit analysis methods, J. Composite Mater., 1 (1967), pp. 366-381.

[56] V. P. Smyshlyaev, V. Nesi, and J. R. Willis, Improved bounds for the yield stress of a model polycrystalline material, J. Mech. Phys. Solids, 48 (2000), pp. 1799-1825.

[57] A. J. M. Spencer, Plasticity theory for fibre-reinforced composites, J. Eng. Math., 26 (1992), pp. $107-118$.

[58] L. Z. Sun AND J. W. JU, Matrix composites containing randomly located and oriented spheroidal particles, J. Appl. Mech.-Trans. ASME, 2004 (2004), pp. 774-785.

[59] P. Suquet, Analyse limite et homogénéisation, C. R. Acad. Sci. Paris Sér. II, 296 (1983), pp. $1355-1358$

[60] P. Suquet, Elements of homogenization for inelastic solid mechanics, in Homogenization Techniques for Composite Media, E. Sanchez-Palencia and A. Zaoui, eds., Lecture Notes in Phys. 272, Springer-Verlag, Berlin, 1987, pp. 193-278.

[61] P. Suquet, Discontinuities and plasticity, in Nonsmooth Mechanics and Applications, J.-J. Moreau and P. D. Panagiotopoulos, eds., Springer-Verlag, Vienna, 1988, pp. 278-340.

[62] P. Suquet, On the overall mechanical behavior of nonlinear composites, C. R. Acad. Sci. Paris Sér. II, 315 (1992), pp. 909-914.

[63] P. Suquet, Overall potentials and extremal surfaces of power law or ideally plastic materials, J. Mech. Phys. Solids, 41 (1993), pp. 981-1002.

[64] D. R. S. TAlBot AND J. R. Willis, Variational principles for inhomogeneous nonlinear media, IMA J. Appl. Math., 35 (1985), pp. 39-54.

[65] L. TARTAR, Compensated compactness and applications to partial differential equations, in Nonlinear Analysis and Mechanics: Heriot-Watt Symposium, Vol. IV, R. Knops, ed., Pitman Res. Notes Math. 39, Pitman, Boston, MA, 1979, pp. 136-212.

[66] L. TARTAR, The compensated compactness method applied to systems of conservation laws, in Systems of Nonlinear Partial Differential Equations, J. M. Ball, ed., Reidel, Dordrecht, The Netherlands, 1983, pp. 263-285.

[67] G. TAYlor, Plastic strains in metals, J. Inst. Metals, 62 (1938), pp. 307-324.

[68] J. R. Willis, The overall elastic response of composite materials, J. Appl. Mech., 50 (1983), pp. 1202-1209.

Copyright $@$ by SIAM. Unauthorized reproduction of this article is prohibited. 